\title{
Diagnosis and Management of Upper Gastrointestinal Neuroendocrine Tumors
}

\author{
Jun Liong Chin ${ }^{1}$ and Dermot $0^{\prime}$ Toole $^{1,2}$ \\ ${ }^{1}$ Department of Gastroenterology, St. Vincent's University Hospital, University College Dublin, Dublin, 'Department of Clinical Medicine, \\ Trinity College Dublin, The University of Dublin, Dublin, Ireland
}

Upper gastrointestinal neuroendocrine tumors (NETs) are rare tumors which are increasingly recognised by practising endoscopists. After confirmation by endoscopic biopsies of these focal lesions, many questions may arise. As NETs are less frequently encountered compared to other malignancies or gastrointestinal pathology, many endoscopists may not fully understand the natural history, diagnosis and management of these tumors. In this review, we aim to update the practising endoscopist on the key clinical features and management of patients with upper gastrointestinal NET. Clin Endosc 2017;50:520-529

Key Words: Esophageal; Gastric; Duodenal; Neuroendocrine tumor

\section{INTRODUCTION}

Upper gastrointestinal neuroendocrine tumors (NETs) are rare tumors which are increasingly diagnosed by endoscopists. They form part of a group of digestive NETs referred to as gastroenteropancreatic NETs, where the overall incidence has dramatically increased worldwide. ${ }^{1,2}$ It is recognised that there appears to be a true increased incidence but incidentally found lesions represent a significant portion of this rise in tumors discovered. Lesions of the stomach, duodenum and rectum have been cited as a strong contributing factor due to the frequency of endoscopy, including screening. ${ }^{1,3,4}$ One large US based population study has recently reported a staggering 15 -fold increase in the incidence of gastric NETs (g-NETs) since the 1970s. ${ }^{4}$

Upper gastrointestinal NETs can be discovered sporadically or rarely at workup for patients with stage 4 diseases. Some cases are also seen as part of inherited syndromes such as

Received: November 9, 2017 Accepted: November 19, 2017

Correspondence: Dermot O’Toole

Department of Clinical Medicine, Trinity College Dublin, The University of Dublin, St James's Hospital, D08 W9RT, Dublin, Ireland

Tel: +353-1-896-2101, Fax: +353-1-454-2043, E-mail: otooled1@tcd.ie

(cc) This is an Open Access article distributed under the terms of the Creative Commons Attribution Non-Commercial License (http://creativecommons.org/ licenses/by-nc/3.0) which permits unrestricted non-commercial use, distribution, and reproduction in any medium, provided the original work is properly cited. multiple endocrine neoplasia type 1 (MEN-1). Commonly, the sporadic form of g-NETs are noted on endoscopy as unusual non-villous type polyps or nodules in the stomach or duodenum. Esophageal NETs tend to have a central ulceration. After confirmation by endoscopic biopsies (reported to stain positive for synaptophysin and chromogranin A), many questions may arise:

- What should we do next?

- Is endoscopic ultrasound (EUS) required to further evaluate these lesions?

- Should we perform an endoscopic resection and if so, what is the best approach?

- Should we refer this patient for surgery?

- Is cross sectional imaging or other investigations required?

- Is a surveillance strategy adequate and if so, at what interval should surveillance gastroscopy be performed?

Many of these questions, may sound familiar to the practising endoscopist.

As NETs are less frequently encountered compared to other malignancies or gastrointestinal pathology, the natural history, diagnosis and management of patients with NETs may not be well understood by all endoscopists. In this review, we endeavour to update the practising endoscopist on the key clinical features and management of patients with upper gastrointestinal NETs. 


\section{ESOPHAGEAL NEUROENDOCRINE TUMORS}

Esophageal NETs are often known as esophageal neuroendocrine carcinoma (NEC) because this tumor is frequently poorly differentiated with a high grade (high mitotic rate, elevated Ki-67, extensive necrosis and nuclear atypia). Two main histological types exist for esophageal NECs, the most common form being small cell esophageal NEC (close to 90\%) versus the large cell type. Hence, this tumor is also known in the literature as small cell carcinoma of the oesophagus.

Esophageal NECs have been reported to represent $0.3 \%$ to $3.8 \%$ of all esophageal carcinomas in the east ${ }^{5-8}$ and west. ${ }^{9-}$

${ }^{11}$ According to Surveillance, Epidemiology and End Results Program (SEER), esophageal carcinoma has an age adjusted incidence rate of 4.2 per 100,000 persons per year. Due to the rarity of esophageal NEC, the actual age adjusted incidence rate is not reported for this subtype of tumor.

\section{Clinical and endoscopic features of esophageal neuroendocrine tumors}

Esophageal NECs are aggressive and often found to have metastasised or advanced locally when diagnosed. Therefore, a diagnosis of esophageal NEC portends a poor prognosis. ${ }^{11}$ Most patients present with symptoms similar to more common adenocarcinomas or squamous cell esophageal cancers, such as progressive dysphagia (79\%), weight loss (54\%), and retrosternal or epigastric pain $(46 \%){ }^{12}$

At endoscopy, esophageal NECs are often observed as either a flat or exophytic lesion, frequently with central ulceration., ${ }^{5,13}$ These lesions can also be seen sometimes as multiple nodules (polypoid) and can be large, measuring up to 3 to $6 \mathrm{~cm}$ in size. Esophageal NECs are usually located in the middle or lower third of the oesophagus. ${ }^{5,7}$ EUS should be performed for accurate local staging for esophageal NECs as lymph node metastases are present in almost half of all patients when diagnosed, if passage of the EUS echoendoscope is possible. ${ }^{5,6,14}$ Other imaging modalities which are necessary for staging, include cross sectional computed tomography (CT) and 18F-Fluorodeoxyglucose positron emission tomography $\left({ }^{18} \mathrm{~F}-\mathrm{FDG}\right.$ PET)/CT. Due to the absence of somatostatin receptors in poorly differentiated esophageal NEC, the role of somatostatin receptor imaging (e.g., using ${ }^{111}$ Indium Octreotide scintigraphy or ${ }^{68}$ Gallium-DOTATOC/DOTATATE PET/CT) is limited.

\section{Management of esophageal neuroendocrine tumors}

Treatment of esophageal NECs are largely stage dependent and principles for treatment are derived from algorithms used for small cell carcinoma of the lung. In limited stage or localised disease (negative lymph nodes), surgical resection has been shown to be effective, compared to chemotherapy alone or chemoradiation. ${ }^{15}$ However, the overall survival after oesophagectomy was reported to be $52 \%$ at 3 years (median survival of 44.9 months). Endoscopic resection has also been reported in tumors $<2 \mathrm{~cm}$ but data for this is limited. ${ }^{6}$ In locoregional disease (positive node), treatment with chemoradiation can only achieve an overall survival of $32 \%$ at 3 years (median survival of 17.8 months). ${ }^{15}$ For metastatic disease, the current recommendation is platinum-based chemotherapy, usually in the form of cisplatin and etoposide as a first line agent. Esophageal NEC patients with metastatic disease treated with chemotherapy alone were found to have an overall survival of only $4 \%$ at 3 years (median survival of 7.8 months). ${ }^{15}$

\section{GASTRIC NEUROENDOCRINE TUMORS}

G-NETs can be classified into three subtypes: ${ }^{16}$ type 1 g-NETs which is the commonest, occur due to hypergastrinaemia in the setting of chronic autoimmune atrophic gastritis; type 2 g-NETs is also due to hypergastrinaemia but this occurs in the setting of gastrin secreting tumors, usually in patients with MEN-1 and as part of a Zollinger Ellison syndrome; in type $3 \mathrm{~g}$-NETS, these tumors are sporadic and usually of high grade with poor differentiation, mimicking gastric carcinoma. Differentiating the subtypes of g-NETs is fundamentally important as this influences the treatment decisions. ${ }^{17-19}$ Understanding that almost all type 1 g-NETs behaves in a rather indolent manner is reassuring while needing to be aggressive with type $3 \mathrm{~g}$-NETs as these tumors spread early is crucial.

The annual age-adjusted incidence of g-NETs is estimated to be around 0.2 per 100,000 in Europe ${ }^{18}$ and has been reported to be around 0.3 per 100,000 in the United States. ${ }^{1}$ G-NETs represent approximately $4.6 \%$ of all NETs, according to SEER. ${ }^{2}$ In one prospective study, g-NETs was reported to represent close to a quarter of all digestive NETs during the study year, ${ }^{20}$ but this figure may vary from country to country and also depends on ethnic demography. ${ }^{1,20-22}$

\section{Clinical and endoscopic features of type 1 gastric neuroendocrine tumors}

Patients with type $1 \mathrm{~g}$-NETs are most commonly encountered, estimated to be close to $70 \%-80 \%$ of all g-NETs, with a female preponderance. Type $1 \mathrm{~g}$-NETs are associated with chronic atrophic gastritis (autoimmune destruction of gastric parietal cells) and auto-antibodies to either intrinsic factor or parietal cells (rarely both), also described as pernicious anae- 

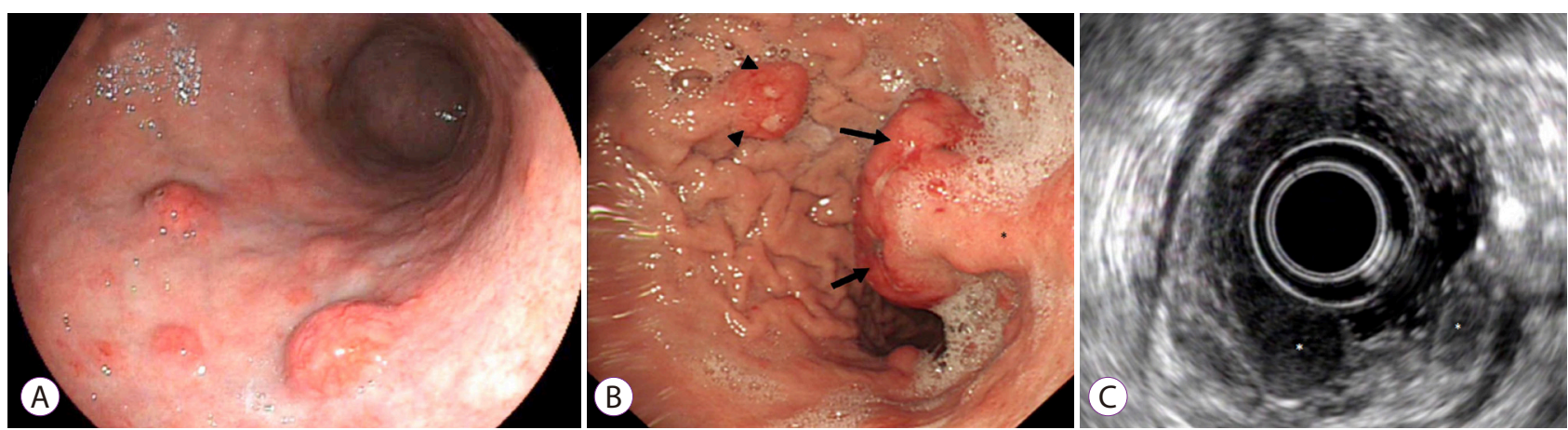

Fig. 1. (A) Classical type 1 gastric neuroendocrine tumors (g-NETs) or neoplasms with marked surrounding gastric mucosal atrophy, evident by loss of gastric folds. (B) Multiple type $1 \mathrm{~g}$-NETs, larger $27 \mathrm{~mm}$ lesion (denoted by black arrows) and smaller $12 \mathrm{~mm}$ lesion (indicated by black arrowheads). Endoscopic ultrasound (EUS) showed large $27 \mathrm{~mm}$ g-NET was within submucosal layer but lymph nodes were pathologically enlarged, uT1N1. (C) EUS appearance of two small g-NETs (*); the larger measures $9 \mathrm{~mm}$ and smaller $6 \mathrm{~mm}$ in maximum dimensions and are seen to lie in the mucosa and submucosal compartments.

mia, leading to gastric achlorhydria and hypergastrinaemia (via loss of feedback mechanism). Hypergastrinaemia results in proliferation of enterochromaffin-like (ECL) cells resulting in neuroendocrine cell hyperplasia and ultimately in clustering of ECL cells into tumorlets (small ECLomas) and eventually the development of type $1 \mathrm{~g}$-NETs.

Patients can be diagnosed with type $1 \mathrm{~g}$-NETs when referred for gastroscopy for anaemia (which can be hypochromic due to reduced iron absorption; normochromic normocytic due to mixed picture or anaemia of chronic disease; or indeed macrocytic anaemia due to reduced B12 absorption), dyspepsia or incidentally discovered. During gastroscopy, small and frequently multiple polyps or nodules $(<1$ to $2 \mathrm{~cm})$ can be observed in the gastric fundus and corpus (Fig. 1A, B). Other endoscopic clues suggesting a diagnosis of type 1 g-NETs include endoscopic appearance of reduced or flattened gastric folds and a low volume gastric acid/mucus pool. Unlike gastric fundal polyps which are also multiple, g-NETs tend to have a rich vascular supply but their colour and shape can vary considerable (flat, sessile, macular, ulcerated when large). Achlorhydria can be confirmed by a high $\mathrm{pH}$ on gastric aspirate during gastroscopy and rarely requires 24hour $\mathrm{pH}$ monitoring. It is imperative to exclude concomitant Helicobacter pylori infection as this may also cause diffuse panatrophic gastritis. EUS examination is recommended for type $1 \mathrm{~g}$-NETs larger than $1 \mathrm{~cm}$ prior to endoscopic resection. Frequently, the findings on EUS are well-demarcated hypoechoiec mucosal/submucosal lesions with regular borders (Fig. 1C). Assessment of enlarged regional lymph nodes can also be performed at EUS survey but they are rarely involved in type $1 \mathrm{~g}$-NETs. Further cross sectional imaging seldom alters the management for type $1 \mathrm{~g}$-NETs and therefore not required unless the lesions are large (predicted $>\mathrm{uT} 1$ or in the presence of nodes); in rare patients with loco-regional or more advanced metastatic cases, axial CT and somatostatin receptor imaging are however mandated. ${ }^{23,24}$
Several laboratory tests are required to confirm the diagnosis of type $1 \mathrm{~g}$-NETs, which include a full blood count to confirm the type of anaemia; an elevated fasting serum gastrin (in the absence of proton pump inhibitor [PPI]); anti-parietal cell and intrinsic factor antibodies (not exclusive); serum B12 and iron studies. ${ }^{19}$ Thyroid dysfunction should also be assessed and monitored as these patients are at risk of developing subclinical or clinical hypothyroidism. Serum chromogranin A can often be helpful, especially for patients with chronic atrophic gastritis as this leads to an increase risk of developing type 1 g-NETs. ${ }^{25}$ However, the utility of serial chromogranin A as a surveillance strategy for disease progression in type 1 g-NETs has not been shown yet.

\section{Clinical and endoscopic features of type 2 gastric neuroendocrine tumors}

Type 2 g-NETs are rare, accounting for only 5\%-6\% of all g-NETs. ${ }^{19}$ Patients with type 2 g-NETs often present for screening gastroscopy after confirmation of an MEN-1 kindred. Occasionally, these patients can present with severe abdominal pain due to multiple gastric and duodenal ulceration; watery diarrhoea due to excessive gastric acid production (described as Zollinger Ellison syndrome), which can be associated with an autosomal dominant MEN-1 syndrome. ${ }^{26}$

Endoscopic features of type $2 \mathrm{~g}$-NETs are similar in appearance to type $1 \mathrm{~g}$-NETs, usually multiple, $<1-2 \mathrm{~cm}$ polypoid lesions. However, in contrast to type $1 \mathrm{~g}-\mathrm{NETs}$, the adjacent gastric folds are significantly hypertrophied with large mucous acid pools, which subtly replenishes at the end of the procedure. Both the gastric and duodenal mucosa showed signs of acid-related damage with occasionally multiple areas of ulceration. It is mandatory to carefully appraised the duodenal cap, bulb and first to third part of duodenum for any raised duodenal polyp(s) (often pale, sessile lesion) which could indicate frequently associated duodenal gastrinoma(s). For patients suspected to have type $2 \mathrm{~g}$-NETs, EUS evaluation 
must be performed for the assessment of any duodenal or pancreatic NETs. ${ }^{27}$ The EUS appearance of type 2 g-NETs is similar to type 1, but the surrounding hypertrophied gastric mucosal folds are clearly evident (Fig. 2). Cross sectional imaging in the form of $\mathrm{CT}$ or magnetic resonance imaging of the abdomen is recommended for type 2 g-NETs associated with MEN-1. Similarly, functional imaging with ${ }^{111}$ Indium Octreotide scintigraphy or ${ }^{68}$ Gallium-DOTATOC/DOTATATE $\mathrm{PET} / \mathrm{CT}$ can be considered in these patients to fully evaluate

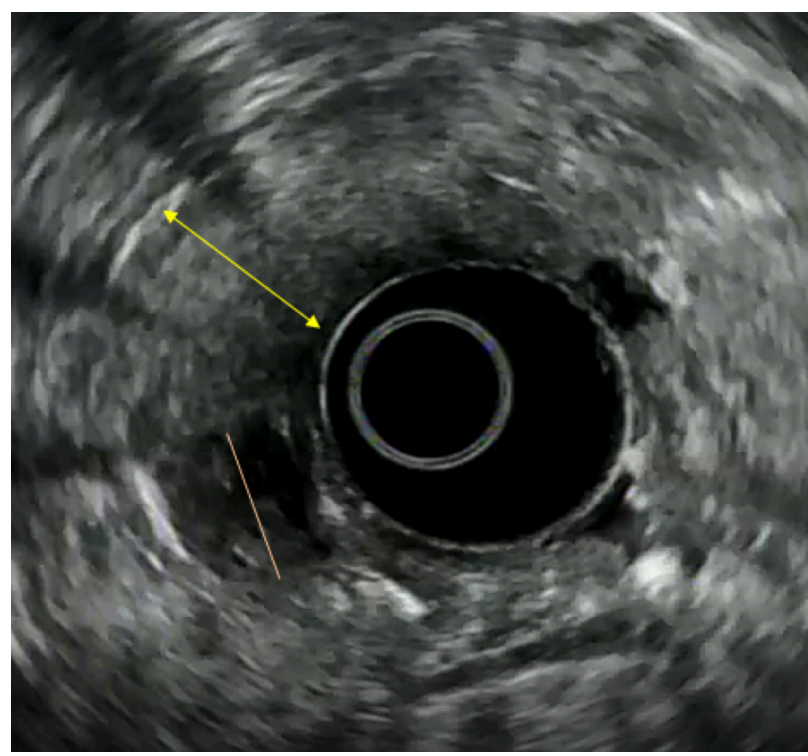

Fig. 2. Type 2 gastric neuroendocrine tumor (g-NET) confined to mucosal layer on endoscopic ultrasound (denoted by orange line), with prominently hypertrophied gastric mucosal layer (indicated by yellow double arrows). This patient also had duodenal gastrinomas and Zollinger Ellison syndrome with multiple pancreatic NETs. and stage the disease burden.

Confirmation of an elevated fasting serum gastrin is of key importance, preferably prior to commencement of PPI therapy. After PPI therapy has been started, it is questionable if weaning PPI therapy is safe at the expense of development of life threatening complication from peptic ulceration. We do not recommend such approach unless clinical suspicion is low. Other laboratory tests as described for type $1 \mathrm{~g}$-NETs serves to exclude this subtype and thereby confirming type 2 g-NETs. Further description for laboratory assessment for type 2 g-NETs associated with MEN-1 will be described later for in duodenal NETs (d-NETs).

\section{Clinical and endoscopic features of type 3 gastric neuroendocrine tumors}

Type $3 \mathrm{~g}$-NETs has been reported to account for almost a quarter of all g-NETs. ${ }^{19}$ Previous distinction of type 3 sporadic solitary NET and type 4 sporadic solitary NEC, ${ }^{28}$ has been supplanted by current understanding and description of well-differentiated versus poorly differentiated type 3 g-NETs. In fact regardless of whether type $3 \mathrm{~g}$-NETs is well or poorly differentiated, most behave in a very aggressive manner and have a high histological grade. Consequently, type 3 g-NETs often present with early involvement of regional lymph nodes or can present with metastatic disease when diagnosed.

These patients may present with symptoms which can be attributed to gastric cancer, such as dyspepsia, weight loss and anaemia, although not infrequently type $3 \mathrm{~g}$-NETs can also be found incidentally when gastroscopy is performed for other indications. On endoscopy, type 3 g-NETs usually occur singly and may be large, up to 2 to $3 \mathrm{~cm}$ in size (Fig. 3). These tumors
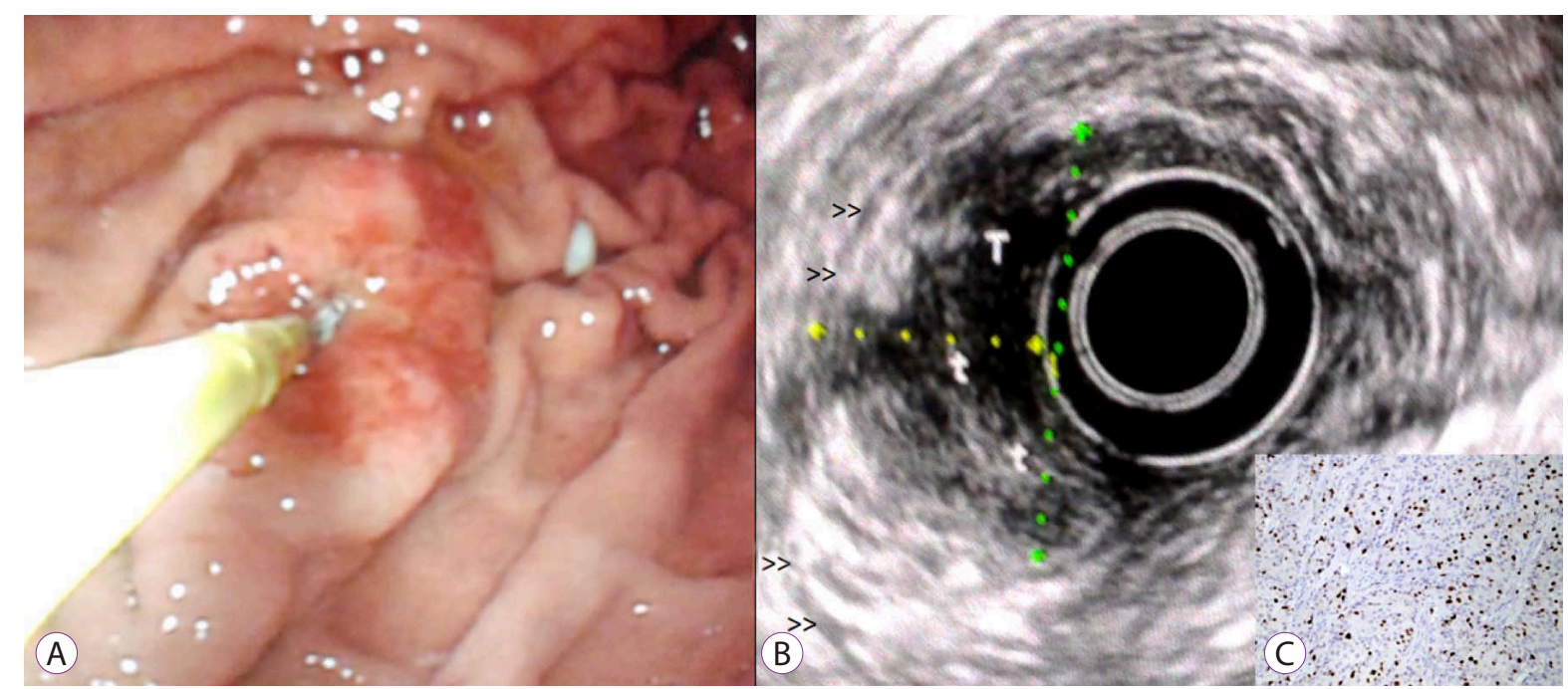

Fig. 3. Example of a type 3 gastric neuroendocrine tumor in distal gastric body. (A) Endoscopically the lesion is quite large over $2.5 \mathrm{~cm}$ and is sessile with a broad, fixed base and a central depressed region. (B) At endoscopic ultrasound, the lesion can be seen to extend to touch the deep muscle layer (double arrow heads) and was predicted uT2 (N0) but after surgical resection the final pathological stage was pT2N1 (one single small node involved) with a Ki-67 of 30\% (insert, C, $\times 200$ ). 
are frequently located in the gastric antrum and can be distinguished from gastric adenomas by the absence of surface villiform architecture, which can be enhanced with virtual chromoendoscopy. For the assessment of type 3 g-NETs, EUS examination is mandatory to assess for depth of invasion into the mucosal layers and the presence of lymph nodes in the gastro-hepatic and peri-gastric areas (Fig. 4). Cross sectional imaging with $\mathrm{CT}$ and ${ }^{111}$ Indium octreotide scintigraphy are required to fully stage type $3 \mathrm{~g}$-NETs.

\section{Management of gastric neuroendocrine tumors}

It has been well-recognised that treatment of g-NETs depends on the subtype, even when the disease has metasta-

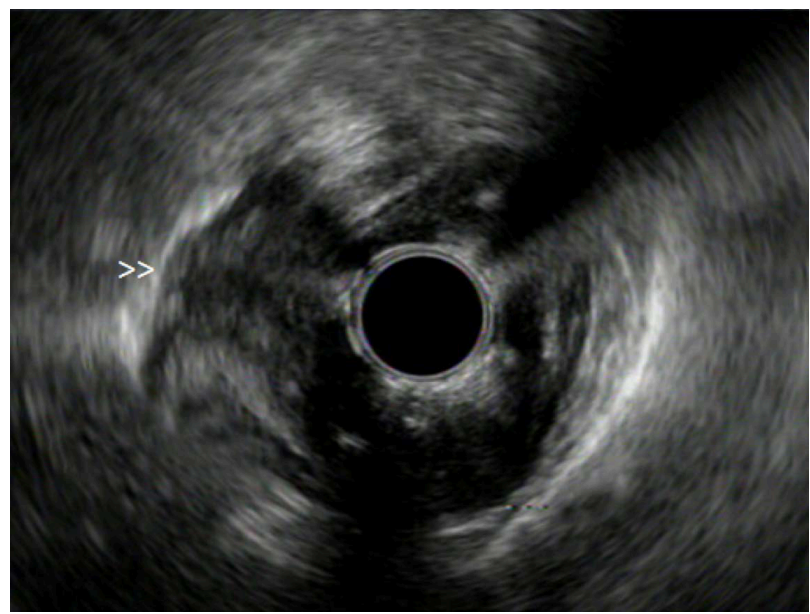

Fig. 4. Endoscopic ultrasound showing a type 3 gastric neuroendocrine carcinoma (NEC) that is bulky on the posterior wall of the gastric antrum and is extending outside the gastric wall (double arrow heads) serosa (muscularis propria indicated). Surgery confirmed an aggressive NEC with a high Ki-67 of $60 \%$ and 4 positive lymph nodes. sized. ${ }^{18,19,29}$ The grade of g-NETs are classified into 3 categories (not to be confused with the subtype) depending on two measures: the mitotic rate reported by the number of mitosis per high power field (hpf) (Grade 1 [G1], $<2$ mitoses/10 hpf; Grade 2 [G2], 2 to 20 mitoses/10 hpf; and Grade 3 [G3], >20 mitoses/10 hpf); and the Ki-67 proliferation index which is described as a percentage (G1, <3\%; G2, $3 \%$ to $20 \%$; and G3, $>20 \%$ ). When both measures are obtained, the higher value is assigned to grade the g-NET for that patient. Moreover, the morphology of g-NETs can frequently help us predict the biology of these tumors. Type $3 \mathrm{~g}$-NETs are commonly poorly differentiated with large cell (sometimes described as NEC) while for type $1 \mathrm{~g}$-NETs, these NET cells are usually well-differentiated with positive immunohistochemistry staining for chromogranin A and synaptophysin (markers of neuroendocrine phenotype). A negative cytokeratin immunohistochemistry can also help differentiate gastric carcinoma from type $3 \mathrm{~g}$-NETs.

Not uncommonly in many cases of g-NETs, gastric mapping biopsies are not done on index gastroscopy to confirm gastric mucosal atrophy or exclude intestinal metaplasia. This is recommended to confirm the diagnosis of type $1 \mathrm{~g}$-NETs and differentiation of different subtypes. ${ }^{19}$ Although type 1 g-NETs have an excellent prognosis and patient survival, this tumor rarely metastasize to regional lymph nodes and liver, close to $8 \%$ in one study. ${ }^{30}$ Similarly, patients with chronic atrophic gastritis also are predisposed to gastric mucosal atrophy and intestinal metaplasia, putting them at debatably higher or uncertain risk of developing gastric adenocarcinomas. The literature from the east would suggest biennial screening gastroscopy for these patients, ${ }^{31}$ but there is a paucity of data in the west. ${ }^{32}$

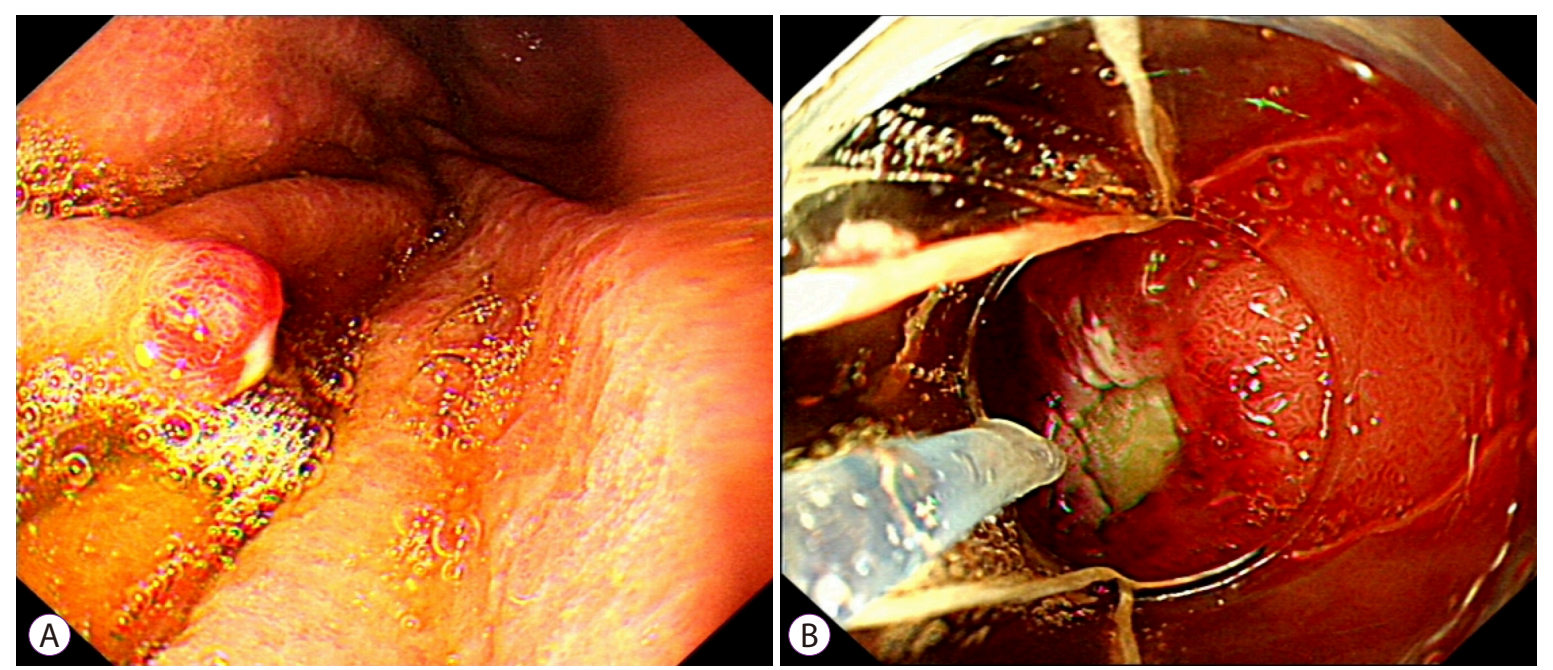

Fig. 5. (A) Small type 1 gastric neuroendocrine tumor approaching $1 \mathrm{~cm}$ in the mid-body greater curve. (B) Endoscopic mucosal resection performed using a cap assisted endoscopic mucosal resection device (Duette ${ }^{\circledR}$ Multi-Band Mucosectomy Device; Cook Medical, Bloomington, IN, USA). 
For type $1 \mathrm{~g}$-NETs, the current recommendation from both European ${ }^{18,19}$ and North American ${ }^{33}$ Neuroendocrine Societies favours a surveillance strategy for small lesions and resection for those approaching $1 \mathrm{~cm}$. Some authors have recommended a more aggressive endoscopic resection policy (resection of all visible lesions) ${ }^{34}$ but this stems from a local retrospective analysis and embarking on endoscopic resection for every tiny lesion observed in cases of type 1 g-NET may also entail some morbidity. At present, there are no current randomised controlled trials (RCT) to suggest which strategy is preferable. Hence, this decision should be individualised, can depend on availability of local expertise or resource and generally, made in partnership with the patient. The current surveillance interval recommended by the European Neuroendocrine Tumor Society is between 1 to 2 years. For type $1 \mathrm{~g}$-NETs $>1 \mathrm{~cm}$, endoscopic resection is widely recommended in Europe ${ }^{18,19}$ This can be performed by cap-assisted endoscopic resection (preferable for multiple g-NETs) (Fig. 5); snare resection after saline lift (or with saline containing 1:10,000 epinephrine); or endoscopic submucosal dissection (ESD). In expert centres practising ESD, ESD for type $1 \mathrm{~g}$-NETs have been reported to be efficacious and safe with similar resection and complication rates to endoscopic mucosal resection (EMR). ${ }^{35-37}$ ESD provides a significantly lower vertical margin excision rate for type 1 g-NETs but this should be balance with operative time and availability.

In North America, guidance for g-NETs of $>1$ to $2 \mathrm{~cm}$ ( $<6$ polyps) includes surveillance within 3 years; endoscopic resection; or resection if EUS showed submucosal involvement. ${ }^{33}$ For type $1 \mathrm{~g}$-NET lesions $>2 \mathrm{~cm}$, a combination of surveillance, endoscopic resection(s) if possible or surgery can be employed. It is important to note that type $1 \mathrm{~g}$-NETs are reported to recur in most patients after endoscopic resection (up to $63 \%$ in a median follow up of 8 months) and hence, surveillance is recommended after index therapy in every 1-2 years. A distinction between recurrence and detection of other lesions as they grow is likely to be more appropriate and this continued surveillance and resection of lesions getting close to $1 \mathrm{~cm}$ is the strategy adopted by the authors.

For type $1 \mathrm{~g}$-NETs that are too large for safe endoscopic resection or predicted T2 (or localised nodal disease), surgical resection is preferable and can vary between limited resection (wedge resection) to more radical oncological resection if nodes are present. Formerly, antrectomy was employed to control gastrin secretion but this has been largely abandoned. ${ }^{19}$

Although RCT data is lacking for somatostatin analogue (SSA) therapy in type $1 \mathrm{~g}$-NETs, SSA treatments can be con- sidered in certain circumstances: ${ }^{19}$ for patients with multiple type 1 tumors which cannot be eradicated with endoscopic therapy (large numbers of tumors); nodal or metastatic disease with proven somatostatin receptor affinity (usually on immunohistochemistry, rather than octreotide scintigraphy due to small size and low uptake) and low Ki-67 index. In limited series, SSA treatment has been shown to regress type $1 \mathrm{~g}^{-\mathrm{NETs}^{38}}$ but recurrence is seen after discontinuation of therapy. ${ }^{39}$

For patients with type 2 g-NETs, treatment is usually dictated by the presence of other NETs in the pancreas and often duodenum, due to its association with MEN-1. Treatment of pancreatic NETs often take precedence over type 2 g-NETs but given the complexity of each individual case, management and treatment planning of these patient requires careful multidisciplinary assessment. ${ }^{17}$ When amenable, type 2 g-NETs can be treated with EMR while large tumors may require surgery (limited resection), which can be performed during resection of pancreatic NETs.

Surgical resection is usually recommended for type 3 g-NETs due to the high proliferative capacity and frequent nodal involvement at the time of clinical presentation. Whether these type 3 g-NETs are well or poorly differentiated, surgery offers the best oncological treatment outcome for localised disease after complete tumor staging with EUS, CT and either somatostatin imaging (if well differentiated) or ${ }^{18}$ F-FDG PET/CT if poorly differentiated.

\section{DUODENAL NEUROENDOCRINE TUMORS}

D-NET accounts for only $2.8 \%$ of all NETs, according to SEER registry. ${ }^{2}$ The annual age adjusted incidence for d-NETs has been reported to be 0.19 per 100,000. ${ }^{1}$ There are many different types of d-NETs reported in the literature. D-NETs can be classified into 5 different tumor types: non-functional d-NETs (although not associated with a specific clinical syndrome, these tumors can produce serotonin or calcitonin); duodenal gangliocytic paragangliomas; high-grade poorly differentiated duodenal NECs; duodenal gastrinomas; and somatostatinomas (the latter two produce gastrin and somatostatin respectively). ${ }^{40}$ Broadly, it is important to note that $90 \%$ of d-NETs are not associated with a functional clinical syndrome and more than $90 \%$ occur in the first or second part of the duodenum. ${ }^{18}$

\section{Clinical and endoscopic features of duodenal neuroendocrine tumors}

The majority of patients with d-NETs are diagnosed 

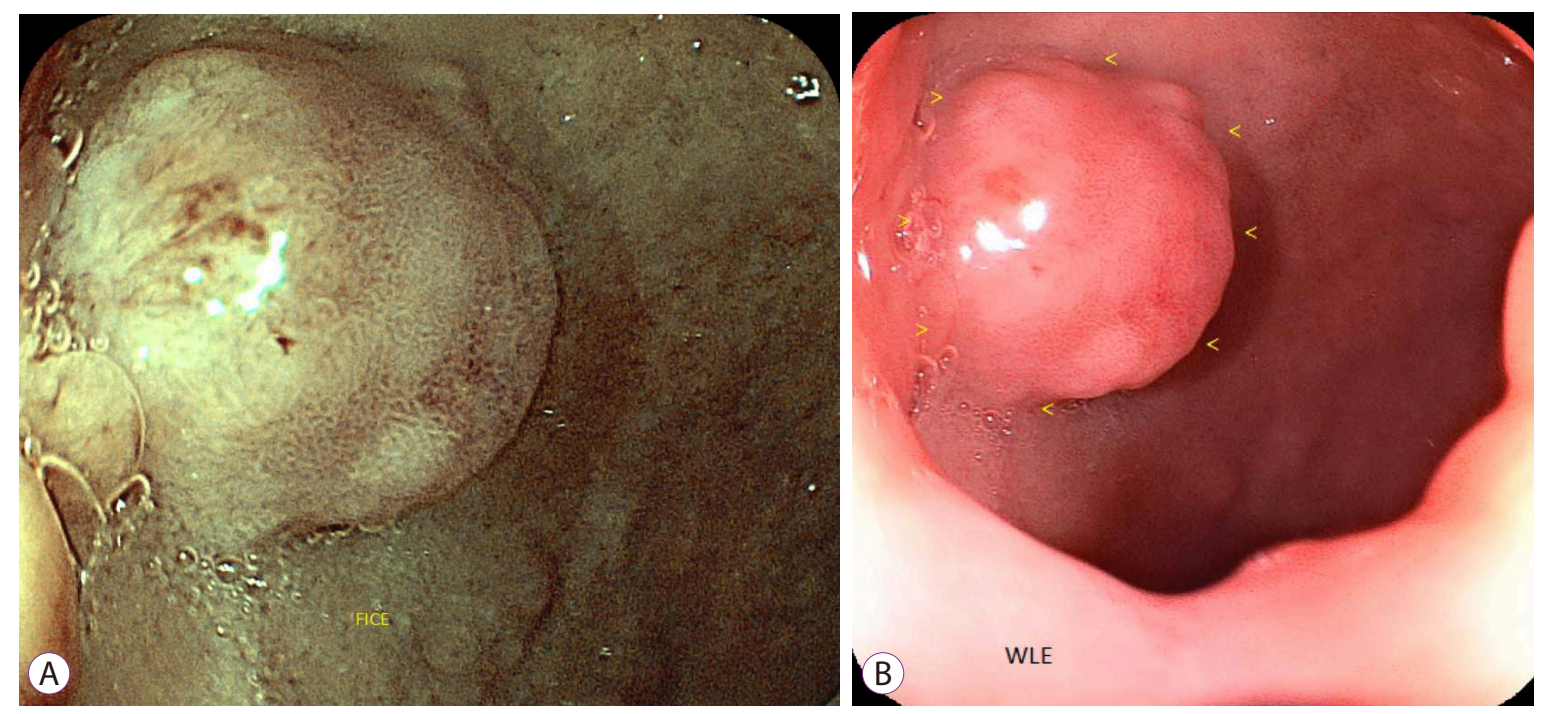

Fig. 6. Sporadic duodenal neuroendocrine tumor in post pyloric position, (A) enhanced by virtual chromoendoscopy (Flexible spectral Imaging Colour Enhancement [FICE]; Fujifilm, Tokyo, Japan) and (B) on white light endoscopy.

incidentally during gastroscopy for other indications, but occasionally can present with symptoms attributed to gastric outlet obstruction when large. Rarely, duodenal gangliotic paragangliomas which occurs in the periampullary region can present with gastrointestinal bleeding, abdominal pain, anaemia or jaundice. ${ }^{18,41}$ Another important association of periampullary d-NETs has also been described in patients with neurofibromatosis type 1 (also known as Von Recklinghausen's disease). ${ }^{42,43}$

On upper gastrointestinal endoscopy, d-NET is typically discovered as single small sessile pale lesion in the duodenal cap or bulb (Fig. 6). The reported mean size of d-NETs are between 1.2 to $1.5 \mathrm{~cm}^{42}$ Close to $10 \%$ of d-NETs can occur as multiple tumors, which should inform the astute endoscopist to suspect a diagnosis of MEN-1. It has been reported that approximately a quarter to a third of patients with d-NETs and Zollinger Ellison syndrome, actually have undiagnosed MEN-1..$^{4,45}$

Confirmation of d-NETs can be achieved with endoscopic forceps biopsy and not infrequently, this can be completely removed if it represents a small sporadic d-NET. For suspicious lesions $>1 \mathrm{~cm}$, confirmation by histology and accurate local staging by EUS are usually recommended first prior to embarking on EMR. As suspicious d-NETs can be diminutive and appear with surrounding gastric heterotopia, accurate description of biopsy site and/or marking must be documented (e.g., anterior wall post-pylorus, roof of duodenal bulb). D-NETs are frequently located in the mucosa and submucosa, but rarely this can extend beyond the submucosa. EUS examination is recommended because d-NETs can be associated with regional lymph node metastases in up to $40 \%-60 \%$ of cases especially in cases of duodenal gastrino-

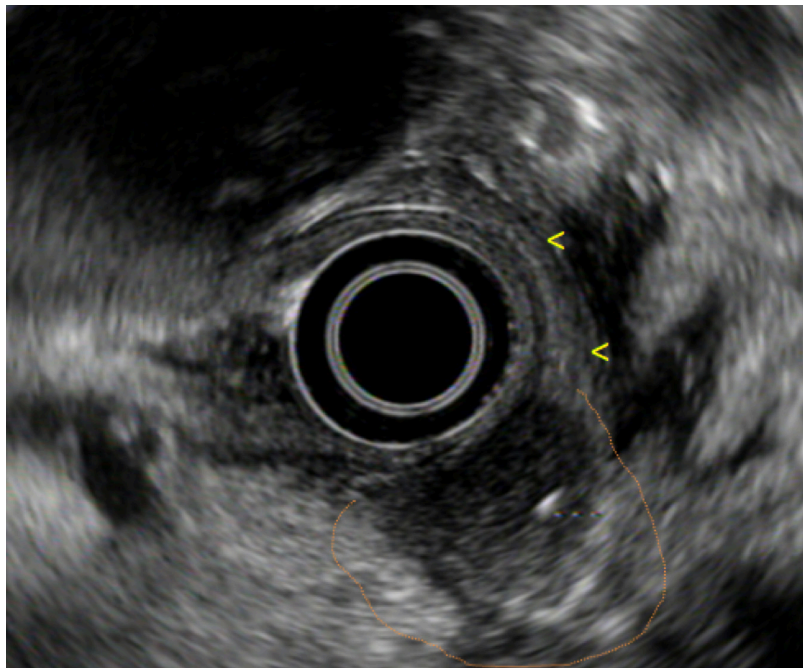

Fig. 7. Duodenal neuroendocrine tumor demonstrated by endoscopic ultrasound to show clear extension beyond the muscularis propria (yellow arrowhead) and therefore, not suitable for endoscopic mucosal resection.

mas. ${ }^{42,46,47}$ Similarly, EUS assesses the degree of submucosal involvement of d-NET and can determine the suitability for endoscopic resection (Fig. 7). Like g-NETs, d-NETs appear as well-demarcated hypoechoiec lesions on EUS. During EUS, careful appraisal of the gastric mucosa for acid-related damage, hypertrophied gastric folds and concomitant g-NETs (previously described in Fig. 2), should be performed, if not previously reported or a clinical suspicion of MEN-1 and Zollinger Ellison syndrome is presented. EUS also allows the pancreas to be fully interrogated for small NETs associated with MEN-1.

Laboratory tests are required for patients newly diagnosed with d-NETs especially important to recognise a potential 
gastrinomas (serum gastrin, chromogranin and occasionally secretin testing). Other laboratory tests (if symptoms are suggestive), include somatostatin, growth hormone releasing factor, and cortisol with urinary 5-hydoxyindoleacetic acid. ${ }^{18}$ For d-NET patients suspicious to have a clinical diagnosis of MEN-1 or a possible family history, we generally performed serum ionised calcium and parathyroid hormone first as routinely available (in conjunction with chromogranin A and gastrin), followed by prolactin; glucagon; insulin with associated fasting glucose; pancreatic polypeptide; and vasoactive intestinal peptide. ${ }^{48}$ Germline testing for MEN-1 should be considered for patients with d-NET, only after appropriate genetic counselling and initial laboratory tests indicating high likelihood for MEN-1. Germline testing allows confirmation of MEN-1 and enables family screening for a specific MEN-1 related mutation. For patients with d-NETs and neurofibromatosis type 1 (Von Recklinghausen's disease), serum somatostatin and calcitonin measurements have been suggested because of somatostatin immunoreactivity in these d-NETs. ${ }^{18}$

\section{Management of duodenal neuroendocrine tumors}

All sporadic d-NETs should be considered for resection, unless medical co-morbidities preclude any improvement in overall patient survival; or in the presence of distant metastases. Endoscopic resection can be performed on small non-gastrinoma d-NETs, after excluding lymph node metastases by EUS or other tumor localisation studies. ${ }^{49} \mathrm{~A}$ variety of endoscopic resection techniques have been described, ${ }^{50}$ which includes: EMR, EMR with ligation device; EMR after circumferential pre-cutting with needle knife; and ESD. Although limited data suggest better pathologically confirmed resection with $\mathrm{ESD}^{3,55}{ }^{350}$ this may not be widely available in many centres and few reports have emerged with regards to substantially higher risk of perforation with ESD for d-NETs. ${ }^{51,52}$ We preferentially utilise standard EMR technique (resection with snare electrocautery after saline lift) when resecting d-NETs. Surgery (local resection with lymphadenectomy or pancreaticoduodenectomy) is usually employed for patients with duodenal gastrinomas or large d-NETs (usually $>2 \mathrm{~cm}$ ); d-NETs invading beyond the submucosa; lymph node metastases; or d-NET in the peri-ampullary region. ${ }^{18}$ Rarely, d-NET with potentially resectable hepatic metastases but without other distant sites of metastases, can be considered for surgery and/or ablative therapy.

Surveillance after resection of d-NETs can depend on many factors, such as local treatment protocol, resources available and accessibility of certain imaging modalities especially outside tertiary centres. Generally, surveillance by upper gastrointestinal endoscopy is recommended ev- ery 2 years after resection of d-NET but this interval has yet to be validated. ${ }^{19}$ After complete endoscopic resection of non-functional d-NETs, follow up investigations in the form of endoscopy examination, plasma chromogranin A and abdominal ultrasound or multi-slice CT, have been recommended at 6, 24 and 36 months. ${ }^{18}$ Likewise, after surgical resection of d-NET, multi-slice CT, octreotide scintigraphy and plasma chromogranin A are recommended at 6 and 12 months, then yearly for at least 3 years. Caution should be placed on interpreting close follow up CT imaging after EMR and surgical resection, as occasionally reactive lymph nodes can be over emphasised.

For d-NETs associated with a functional syndrome $(<10 \%$ of patients), appropriate specific therapy for control of hormone excess state should be commenced, such as: SSA therapy for carcinoid syndrome; and PPI treatment for Zollinger Ellison syndrome. Care of patients with functional d-NETs are now often referred to specialist Neuroendocrine Tumor board or teams.

\section{CONCLUSIONS}

We summarised and highlighted the important clini$\mathrm{cal}$ and management strategies for the endoscopist when confronted with upper gastrointestinal NETs in practice. As NETs are increasingly diagnosed, further evidence will become available to optimise and support our current approach in managing patients with upper gastrointestinal NETs. Endoscopy and endoscopic-related therapy remain the cornerstone for the diagnosis, evaluation and treatment of upper gastrointestinal NETs.

\section{Conflicts of Interest}

The authors have no financial conflicts of interest.

\section{REFERENCES}

1. Yao JC, Hassan M, Phan A, et al. One hundred years after "carcinoid": epidemiology of and prognostic factors for neuroendocrine tumors in 35,825 cases in the United States. J Clin Oncol 2008;26:3063-3072.

2. Modlin IM, Lye KD, Kidd M. A 5-decade analysis of 13,715 carcinoid tumors. Cancer 2003;97:934-959.

3. Modlin IM, Lye KD, Kidd M. A 50-year analysis of 562 gastric carcinoids: small tumor or larger problem? Am J Gastroenterol 2004;99:2332.

4. Dasari A, Shen C, Halperin D, et al. Trends in the incidence, prevalence, and survival outcomes in patients with neuroendocrine tumors in the United States. JAMA Oncol 2017;3:1335-1342.

5. Huang Q, Wu H, Nie L, et al. Primary high-grade neuroendocrine carcinoma of the esophagus: a clinicopathologic and immunohistochemical study of 42 resection cases. Am J Surg Pathol 2013;37:467-483.

6. Lee CG, Lim YJ, Park SJ, et al. The clinical features and treatment modality of esophageal neuroendocrine tumors: a multicenter study in 
Korea. BMC Cancer 2014;14:569.

7. Babu Kanakasetty G, Dasappa L, Lakshmaiah KC, et al. Clinicopathological profile of pure neuroendocrine neoplasms of the esophagus: a South Indian center experience. J Oncol 2016;2016:2402417.

8. Lv J, Liang J, Wang J, et al. Primary small cell carcinoma of the esophagus. J Thorac Oncol 2008;3:1460-1465.

9. Bennouna J, Bardet E, Deguiral P, Douillard JY. Small cell carcinoma of the esophagus: analysis of 10 cases and review of the published data. Am J Clin Oncol 2000;23:455-459.

10. Ku GY, Minsky BD, Rusch VW, Bains M, Kelsen DP, Ilson DH. Smallcell carcinoma of the esophagus and gastroesophageal junction: review of the Memorial Sloan-Kettering experience. Ann Oncol 2008;19:533537.

11. Kukar M, Groman A, Malhotra U, et al. Small cell carcinoma of the esophagus: a SEER database analysis. Ann Surg Oncol 2013;20:42394244.

12. Vos B, Rozema T, Miller RC, et al. Small cell carcinoma of the esophagus: a multicentre rare cancer network study. Dis Esophagus 2011;24:258-264.

13. Egashira A, Morita M, Kumagai R, et al. Neuroendocrine carcinoma of the esophagus: clinicopathological and immunohistochemical features of 14 cases. PLoS One 2017;12:e0173501.

14. Deng HY, Ni PZ, Wang YC, Wang WP, Chen LQ. Neuroendocrine carcinoma of the esophagus: clinical characteristics and prognostic evaluation of 49 cases with surgical resection. J Thorac Dis 2016;8:1250-1256.

15. Wong AT, Shao M, Rineer J, Osborn V, Schwartz D, Schreiber D. Treatment and survival outcomes of small cell carcinoma of the esophagus: an analysis of the national cancer data base. Dis Esophagus 2017;30:1-5.

16. Rindi G, Luinetti O, Cornaggia M, Capella C, Solcia E. Three subtypes of gastric argyrophil carcinoid and the gastric neuroendocrine carcinoma: a clinicopathologic study. Gastroenterology 1993;104:994-1006.

17. O’Toole D, Delle Fave G, Jensen RT. Gastric and duodenal neuroendocrine tumours. Best Pract Res Clin Gastroenterol 2012;26:719-735.

18. Delle Fave G, Kwekkeboom DJ, Van Cutsem E, et al. ENETS consensus guidelines for the management of patients with gastroduodenal neoplasms. Neuroendocrinology 2012;95:74-87.

19. Delle Fave G, O'Toole D, Sundin A, et al. ENETS consensus guidelines update for gastroduodenal neuroendocrine neoplasms. Neuroendocrinology 2016;103:119-124

20. Niederle MB, Hackl M, Kaserer K, Niederle B. Gastroenteropancreatic neuroendocrine tumours: the current incidence and staging based on the WHO and European neuroendocrine tumour society classification: an analysis based on prospectively collected parameters. Endocr Relat Cancer 2010;17:909-918.

21. Tsai HJ, Wu CC, Tsai CR, Lin SF, Chen LT, Chang JS. The epidemiology of neuroendocrine tumors in Taiwan: a nation-wide cancer registry-based study. PLoS One 2013;8:e62487.

22. Hallet J, Law CH, Cukier M, Saskin R, Liu N, Singh S. Exploring the rising incidence of neuroendocrine tumors: a population-based analysis of epidemiology, metastatic presentation, and outcomes. Cancer 2015;121:589-597.

23. Pavel M, de Herder WW. ENETS consensus guidelines for the standard of care in neuroendocrine tumors. Neuroendocrinology 2017;105:193195.

24. Sundin A, Arnold R, Baudin E, et al. ENETS consensus guidelines for the standards of care in neuroendocrine tumors: radiological, nuclear medicine \& hybrid imaging. Neuroendocrinology 2017;105:212-244.

25. Campana D, Ravizza D, Ferolla P, et al. Risk factors of type 1 gastric neuroendocrine neoplasia in patients with chronic atrophic gastritis. A retrospective, multicentre study. Endocrine 2017;56:633-638.

26. Roy PK, Venzon DJ, Shojamanesh H, et al. Zollinger-Ellison syndrome. Clinical presentation in 261 patients. Medicine (Baltimore) 2000;79:379411.

27. O'Toole D, Palazzo L. Endoscopy and endoscopic ultrasound in assessing and managing neuroendocrine neoplasms. Front Horm Res
2015;44:88-103

28. Rindi G, Arnold R, Bosman FT. Nomenclature and classification of neuroendocrine neoplasms of the digestive system. In: Bosman TF, Carneiro F, Hruban RH, eds. WHO classification of tumours of the digestive system 4th ed. Lyon: International Agency for Research on Cancer (IARC); 2010. p. 13.

29. Schindl M, Kaserer K, Niederle B. Treatment of gastric neuroendocrine tumors: the necessity of a type-adapted treatment. Arch Surg 2001;136:49-54.

30. Borch K, Ahrén B, Ahlman H, Falkmer S, Granérus G, Grimelius L. Gastric carcinoids: biologic behavior and prognosis after differentiated treatment in relation to type. Ann Surg 2005;242:64-73.

31. Park YH, Kim N. Review of atrophic gastritis and intestinal metaplasia as a premalignant lesion of gastric cancer. J Cancer Prev 2015;20:25-40.

32. O'Connor A, McNamara D, O'Moráin CA. Surveillance of gastric intestinal metaplasia for the prevention of gastric cancer. Cochrane Database Syst Rev 2013:CD009322.

33. Kunz PL, Reidy-Lagunes D, Anthony LB, et al. Consensus guidelines for the management and treatment of neuroendocrine tumors. Pancreas 2013;42:557-577.

34. Merola E, Sbrozzi-Vanni A, Panzuto F, et al. Type I gastric carcinoids: a prospective study on endoscopic management and recurrence rate. Neuroendocrinology 2012;95:207-213.

35. Li QL, Zhang YQ, Chen WF, et al. Endoscopic submucosal dissection for foregut neuroendocrine tumors: an initial study. World J Gastroenterol 2012;18:5799-5806.

36. Sato Y, Takeuchi M, Hashimoto S, et al. Usefulness of endoscopic submucosal dissection for type I gastric carcinoid tumors compared with endoscopic mucosal resection. Hepatogastroenterology 2013;60:15241529 .

37. Kim HH, Kim GH, Kim JH, Choi MG, Song GA, Kim SE. The efficacy of endoscopic submucosal dissection of type I gastric carcinoid tumors compared with conventional endoscopic mucosal resection. Gastroenterol Res Pract 2014;2014:253860.

38. Campana D, Nori F, Pezzilli R, et al. Gastric endocrine tumors type I: treatment with long-acting somatostatin analogs. Endocr Relat Cancer 2008;15:337-342.

39. Jianu CS, Fossmark R, Syversen U, Hauso Ø, Fykse V, Waldum HL. Fiveyear follow-up of patients treated for 1 year with octreotide long-acting release for enterochromaffin-like cell carcinoids. Scand J Gastroenterol 2011;46:456-463.

40. Klöppel G, Perren A, Heitz PU. The gastroenteropancreatic neuroendocrine cell system and its tumors: the WHO classification. Ann N Y Acad Sci 2004;1014:13-27.

41. Okubo Y, Yokose T, Motohashi O, et al. Duodenal rare neuroendocrine tumor: clinicopathological characteristics of patients with gangliocytic paraganglioma. Gastroenterol Res Pract 2016;2016:5257312.

42. Hoffmann KM, Furukawa M, Jensen RT. Duodenal neuroendocrine tumors: classification, functional syndromes, diagnosis and medical treatment. Best Pract Res Clin Gastroenterol 2005;19:675-697.

43. Stamm B, Hedinger CE, Saremaslani P. Duodenal and ampullary carcinoid tumors. A report of 12 cases with pathological characteristics, polypeptide content and relation to the MEN I syndrome and von Recklinghausen's disease (neurofibromatosis). Virchows Arch A Pathol Anat Histopathol 1986;408:475-489.

44. Benya RV, Metz DC, Venzon DJ, et al. Zollinger-Ellison syndrome can be the initial endocrine manifestation in patients with multiple endocrine neoplasia-type I. Am J Med 1994;97:436-444.

45. Gibril F, Schumann M, Pace A, Jensen RT. Multiple endocrine neoplasia type 1 and Zollinger-Ellison syndrome: a prospective study of 107 cases and comparison with 1009 cases from the literature. Medicine (Baltimore) 2004;83:43-83.

46. Yu F, Venzon DJ, Serrano J, et al. Prospective study of the clinical course, prognostic factors, causes of death, and survival in patients with long-standing Zollinger-Ellison syndrome. J Clin Oncol 1999;17:615-630. 
47. Weber HC, Venzon DJ, Lin JT, et al. Determinants of metastatic rate and survival in patients with Zollinger-Ellison syndrome: a prospective long-term study. Gastroenterology 1995;108:1637-1649.

48. Thakker RV, Newey PJ, Walls GV, et al. Clinical practice guidelines for multiple endocrine neoplasia type 1 (MEN1). J Clin Endocrinol Metab 2012;97:2990-3011.

49. Dalenbäck J, Havel G. Local endoscopic removal of duodenal carcinoid tumors. Endoscopy 2004;36:651-655.

50. Kim GH, Kim JI, Jeon SW, et al. Endoscopic resection for duodenal carcinoid tumors: a multicenter, retrospective study. J Gastroenterol Hepatol 2014;29:318-324.

51. Matsumoto S, Miyatani H, Yoshida Y, Nokubi M. Duodenal carcinoid tumors: 5 cases treated by endoscopic submucosal dissection. Gastrointest Endosc 2011;74:1152-1156.

52. Suzuki S, Ishii N, Uemura M, et al. Endoscopic submucosal dissection (ESD) for gastrointestinal carcinoid tumors. Surg Endosc 2012;26:759763. 